\title{
IMPROVING THE DESIGN PROCESS FOR FACTORIES: MODELLING HUMAN PERFORMANCE VARIATION
}

\author{
Dr. Steve Mason, Professor Tim Baines*, \\ Professor John M. Kay and John Ladbrook \\ Cranfield University, UK \\ *t.s.baines@cranfield.ac.uk
}

\begin{abstract}
:
The process of manufacturing system design frequently includes modelling and, usually, this means applying a technique such as Discrete Event Simulation (DES). However, the computer tools currently available to apply this technique enable only a superficial representation of the people that operate within systems. This is a serious limitation as the performance of people remain central to the competitiveness of many manufacturing enterprises. Therefore, this paper explores the use of probability density functions to represent the variation of worker activity times within DES models.
\end{abstract}

Keywords: Simulation, Human Performance Modelling, Human Performance Variation

\section{INTRODUCTION}

In a manufacturing system design context, the term modelling usually means applying a technique such as computer based Discrete Event Simulation (DES). However, Human Performance Variation (HPV) is often modelled incorrectly in DES models. As a consequence, simulation results can be distorted, providing only a coarse prediction of how people-centered manufacturing systems are likely to perform in practice. This limitation on the ability to adequately model the behavior of people in manufacturing systems is a significant issue. Hence, there is an important need to expand the capability of modelling to include accurate representations of Human Performance Variation.

The research described in this paper has focused on the development of Probability Density Functions to represent the variation of worker activity times within DES models. The paper describes how, contrary to prior evidence in the literature, empirical worker performance data is best represented using a Pearson Type IV distribution. This is demonstrated in a number of simulation experiments which also illustrate that, under certain conditions, HPV can have a significant effect on overall system performance. Indeed, these results indicate that practitioners should always consider testing for sensitivity to HPV.
The paper is structured to first illustrate the research challenge in greater detail, with section 3 then describing the research approach taken. Following this, section 4 explores the nature of HPV in a real production facility. Section 5 describes how this variation can be represented statistically and section 6 then demonstrates the impact of incorporating this variation within simulation models of the facility. The final sections give our conclusions and identify further work.

\section{BACKGROUND}

International market pressures and technical innovation translate into ever increasing demands for wider product variety, mass customization, faster delivery, improved quality and reduced cost [1]. To fulfill these demands, enterprises must continually improve their product design and manufacturing capabilities [2, 3, 4, 5]. Some manufacturing system re-design can take place incrementally, using techniques such as Kaizen [6]. On the other hand, major and radical re-designs are sometimes called for when, for example, a new product line is introduced or a new facility is proposed [7]. Here, a more formal process of manufacturing system design is usually followed.

Modelling is undoubtedly a popular and valuable aid during this process of system design [8, 9]. Usually, modelling is carried out using a computer based tool, the principles of which, are based on the technique of Discrete Event Simulation (DES) [10]. There are a wide variety of tools of this nature and, predominantly, they are used mainly to model the elements of a manufacturing system that are highly deterministic in their behavior (eg: mechanical equipment such as machines, conveyors and robotics) [11]. However, as the elements of a manufacturing system become less deterministic the validity of models rely greater on the skills of the practitioner [12]. In this situation practitioners must be highly skilled in data collection techniques, statistical analysis methods, and also highly knowledgeable about the behavior of the element under consideration [13]. One such issue occurs when attempting to model the behavior of production workers [14, 15]. 
Currently computer tools, based on the technique of DES, treat production people as a simple element that has limited availability [11, 16, 17, 18]. In a typical simulation model 'labor' is defined as a resource that is required by activities such as 'machining' and 'assembly' [7, 11]. Then, by varying the general availability and priorities of the labor resource, the model is used to determine the required number of workers [7, 19]. Occasionally, standard statistical distributions (eg: normal distribution) are used to introduce some variance to the availability of the 'worker resource' and, in this way, some assessment of model sensitivity can be carried out [20]. However, the validity of using such distributions is highly questionable.

Human Performance Variation (HPV) in manual tasks has been studied since the work of Taylor in the late nineteenth century [21]. The possibility that activity duration can be characterised by a Probability Density Function (PDF) has been known for some time [22]. For example, the positively skewed nature of the distribution of activity duration was noted by Conrad in 1954 [23] and this has been confirmed in many studies since [e.g. 22, 24, 25, 26, 27, 28]. However, the exact form of the response has not previously been identified and, so, a number of PDF forms are sometimes used to represent HPV. This is frequently unsatisfactory, for example, Knott and Sury [22] found that:

"A goodness of fit, $X^{2}$ test, was used to test the actual worker time distributions against the lognormal, gamma and beta distributions, none of which proved to be statistically significant."

Judging by the apparent paucity of literature since 1987, this issue has not been studied in depth since the late 1980's. Consequently a variety of standard distributions are still used to estimate HPV [9]. It is therefore clear that a definitive description of the likely form and impact of HPV is needed [29, 30, $31]$ and this therefore is the purpose of the research described here.

\section{RESEARCH METHODOLOGY}

The aim of the research has been to determine a valid method of representing HPV in computer tools, based on the technique of DES, and to assess the impact of this on a typical model of manufacturing systems. To achieve this aim three objectives were identified, namely to:

- Measure the extent of HPV within an actual manufacturing system.

- Develop a statistical representation of this real HPV.

- Include this actual HPV within a typical DES model and evaluate the impact.
A three stage research methodology was defined to complement these objectives and this provides the structure for the remainder of this paper.

\section{HUMAN PERFORMANCE VARIATION: A DEFINITION AND ASSESSMENT}

Human performance within a manufacturing system can be measured in many ways. For example, in terms of how quickly a task is completed; how many task cycles are completed; and the reliability with which a task is completed. The selection of the appropriate measure depends on the objective of the measurement. For people who are directly involved in production (sometimes referred to as direct workers) the duration of the time spent on a discrete activity (eg: fitting a water pump to an engine) is a most valuable measure. Hence, in the context of the work described in this paper Human Performance Variation (HPV) is defined as:

The variation in the time taken to complete a task by a direct worker under normal working conditions.

The term 'normal working conditions' implies that both the operation of the production system in which the worker operates, and worker performance itself, is within the accepted limits for the task [32]. This definition eliminates performance during periods where system perturbations are present, such as when quality problems occur, or where the worker displays unusual behaviors.

With this definition in mind, a detailed study of actual HPV was designed and executed by the authors. An automated activity time measurement system was installed on a real manufacturing system (this is described in detail in [33]). The system was a large-scale, high-precision, highvalue automotive assembly line. Ten manual task operations were monitored over a twelve week period, resulting in a dataset for each operation of the order of 200,000 activity times.

Within the assembly line, each operation was performed by a team of operators who work on a number of different activities throughout the day, spending an hour at a time on each operation, and then rotating to the next. This job rotation helped to reduce monotony and boredom [34]. The data sets gathered were for individual machines operated by the team, however, the researchers were prevented from directly relating activity time variations to specific workers. Similarly, no observations could be made of particular workers over long periods of time. This limitation is discussed further in section 6. 
Examination of the resulting datasets reveals a number of interesting characteristics. Figure 1 shows a sample time series plot of a 12 hour period of activity time data from one operation. It can be seen that each operator achieves a different level of performance in terms of the mean and range of their activity time. This is consistent with the general view in the literature that individual differences do affect worker performance [35].

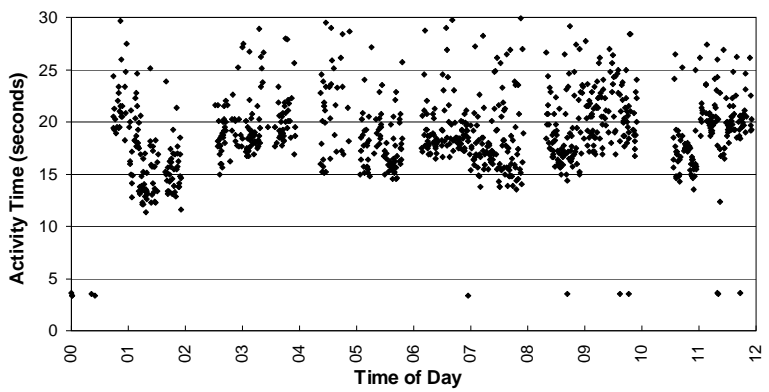

Figure 1: Sample 12 hour time series plot of activity time data for one operation.

Plotting the data as a histogram for each operation (a technique used by numerous researchers [22, 24, $25,26,36]$ ) provides intriguing results as shown in figure 2. Here, the ten curves (one for each operation) have markedly different mean values. This is an unexpected result as the assembly line under study has undergone a rigorous design process to ensure that it is balanced. This would imply that these curves should be similar.

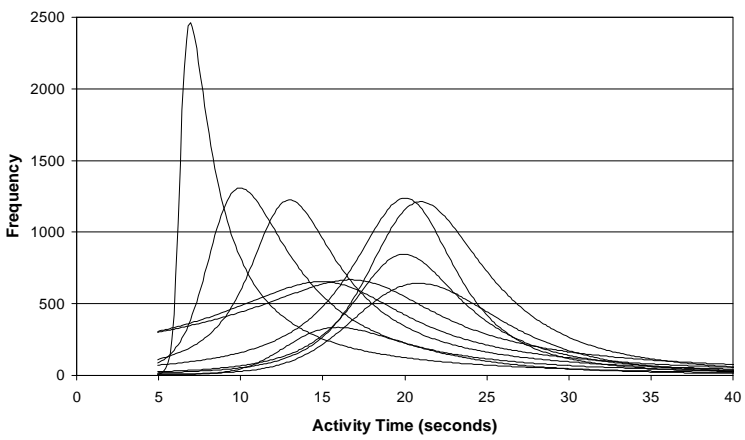

Figure 2: Activity time distributions for the ten operations studied

Closer examination of each distribution also reveals that they are bimodal in nature (Figure 3). The peaks may be due to individual workers having different ways of completing the task, or to the effect of "blocked conditions" where an operator is prevented from passing their completed work onto the next operation because the inter-process buffer is full.

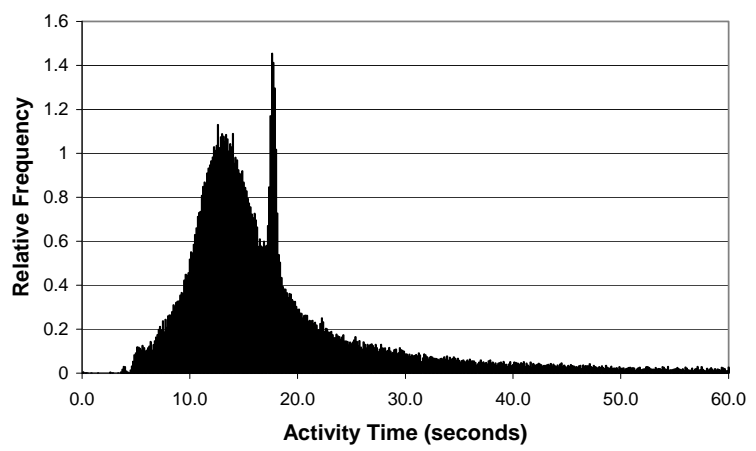

Figure 3: Relative frequency histogram for one operation

The bimodality and skew of the empirical distribution suggests the hypothesis that HPV, in real world production situations, occurs in a number of different forms. These reflect different worker behaviors, ways of working, and other effects. If then, a distribution is created to summaries HPV over a significant time, this may actually be the sum of a number of component distributions. These component distributions may not necessarily themselves be skewed. This then forms a basis for developing a statistical representation of HPV.

\section{DEVELOPING A STATISTICAL REPRESENTATION OF HUMAN PERFORMANCE VARIATION}

The form of the empirical distributions can be estimated by applying curve fitting software. In this case TableCurve2D ${ }^{\mathrm{TM}}$ was used to test the fit of over two thousand different equation forms to the data using least squares estimation. The statistical summary of this process is shown in table 1 . Here, the $r^{2}$ value is shown, for each of the ten production operations assessed in the case study, against the three distributions most commonly used to represent HPV.

\begin{tabular}{|c|c|c|c|c|}
\hline $\begin{array}{c}\text { Operation } \\
\text { Number }\end{array}$ & $\begin{array}{c}\text { Pearson } \\
\text { IV r }\end{array}$ & $\begin{array}{c}\text { Normal } \\
\mathrm{r}^{2}\end{array}$ & $\begin{array}{c}\text { Weibull } \\
\mathrm{r}^{2}\end{array}$ & $\begin{array}{c}\text { Gamma } \\
\mathrm{r}^{2}\end{array}$ \\
\hline 1 & 0.997 & 0.961 & 0.951 & 0.975 \\
\hline 2 & 0.990 & 0.832 & 0.953 & 0.957 \\
\hline 3 & 0.997 & 0.973 & 0.977 & 0.986 \\
\hline 4 & 0.969 & 0.938 & 0.939 & 0.938 \\
\hline 5 & 0.995 & 0.946 & 0.978 & 0.988 \\
\hline 6 & 0.996 & 0.977 & 0.977 & 0.980 \\
\hline 7 & 0.996 & 0.942 & 0.959 & 0.981 \\
\hline 8 & 0.997 & 0.965 & 0.973 & 0.981 \\
\hline 9 & 0.997 & 0.979 & 0.981 & 0.984 \\
\hline 10 & 0.996 & 0.979 & 0.985 & 0.991 \\
\hline
\end{tabular}

Table 1: sample coefficient of determination for least squares fit to activity time data.

The data in Table 1 shows that the curve which gives the most reliable fit, in terms of the $r^{2}$ value across all operations, is the Pearson Type IV. This 
is in contrast to the curves normally cited in the literature as being appropriate to represent HPV, namely; Normal, Exponential [37], Gamma [38], Weibull [39], and the Erlang distribution [40]. Of these the Gamma distribution gives the closest fit.

The activity times, however, differ significantly across the ten operations (figure 2). To make the ranges comparable, and the Pearson Type IV usable in practice, the Coefficient of Variance (CV) needs to be established. The $\mathrm{CV}$ is a measure of dispersion and can be used to compare distributions with different means [22] and is calculated by dividing the standard deviation by the mean. The $\mathrm{CV}$ of worker activity times assumed by researchers in assembly line design varies widely, as shown in table 2. Liu et al. [38] state that, "...high $C V$ values in the order of 0.3 and over are not common occurrence in the real world", although out of curiosity they expanded the CV range in their study from 0.2 to 0.6 . The range of CV values present in a system can drastically alter the design parameters required for effective operation of the system [41, 42].

\begin{tabular}{|l|l|}
\hline \multicolumn{1}{|c|}{ Authors } & $\begin{array}{c}\text { Coefficient } \\
\text { of Variation }\end{array}$ \\
\hline Conrad and Hille (1955) & $0.18-0.32$ \\
\hline Kala and Hitchings (1973) & $0.00-0.24$ \\
\hline Slack and Wild (1980a) & $0.25-0.32$ \\
\hline Knott and Sury (1987) & $0.22-0.57$ \\
\hline Chow (1987) & $0.22-0.47$ \\
\hline Hira and Pandey (1987) & $0.00-1.00$ \\
\hline Dar-El and Mazer (1989) & $0.00-0.30$ \\
\hline Blumenfeld (1990) & $0.00-1.00$ \\
\hline Liu et al (1996) & $0.20-0.60$ \\
\hline
\end{tabular}

Table 2: Coefficient of variation of activity times assumed by authors from 1955 - 1996.

The CV results for the ten operations in the present study range between 0.31 and 0.63 , significantly higher than many researchers have assumed in the past. It is worth noting that the datasets also contain some evidence of trends in performance over time. Using the technique of waveform education attributed by Minors and Waterhouse [43] to Czeisler [44], the underlying oscillatory nature of the data can be seen. By concatenating all the data for an operation onto a 24 hour timescale, and dividing the dataset into hourly time slots, it can be seen that the mean activity time changes over time, as shown in figure 4 . The range in the mean of this trend is 1.86 seconds. Therefore, although the remainder of this paper primarily focuses on assessing the impact of the Person Type IV, some consideration is made of temporal trends on model validity.

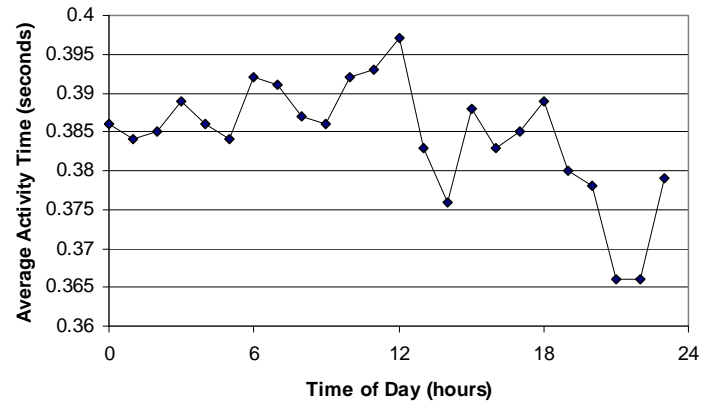

Figure 4: Trend in mean activity time for one operation over 24 hours, three shift system

\section{EXPLORING THE IMPACT OF HPV ON MANUFACTURING SYSTEM MODELS}

This section of the paper explores the impact of incorporating HPV on models of a real manufacturing system. The models chosen are of the system, from which, the data of HPV was collected. Although this approach has some limitations (as discussed later) the size of this facility, and the depth to which the research team understand its operation, mean that it is very well suited to this initial assessment.

One goal in carrying out this study was to assess the relative significance of HPV on the performance of a model. Therefore, a series of five DES models were constructed with each representing a slightly different scenario. A summary of these models is shown in table 3 . Model 0 represents the system with deterministic activity times and no breakdowns. The deterministic output of this model can therefore be considered as the ideal performance of the system. In Model 1 breakdowns are represented stochastically and are based on empirical data from the real system. Comparison of the results of Model 0 and Model 1 gives a measure of the impact of modelling breakdowns on system performance. The activity times remain deterministic.

Model 1 forms the basis for Models 2, 3 and 4 and is focused at assessing HPV. Currently the host organization uses a Normal distribution with the work standard time as the mean and a standard deviation of $8 \%$ of the work standard time. A single PDF technique is implemented in Model 3, and a probability density surface technique is implemented in Model 4 (this allows the temporal trends mentioned earlier to be incorporated). 


\begin{tabular}{|c|l|}
\hline $\begin{array}{c}\text { Model } \\
\text { Name }\end{array}$ & \multicolumn{1}{c|}{ Description } \\
\hline Model 0 & $\begin{array}{l}\text { Deterministic activity time } \\
\text { No stochastic modelling of breakdowns }\end{array}$ \\
\hline Model 1 & $\begin{array}{l}\text { Deterministic activity time } \\
\text { Breakdowns modeled stochastically }\end{array}$ \\
\hline Model 2 & $\begin{array}{l}\text { Stochastic activity time, Normal PDF } \\
\text { Breakdowns modeled stochastically }\end{array}$ \\
\hline Model 3 & $\begin{array}{l}\text { Stochastic activity time, Single Pearson Type } \\
\text { IV PDF } \\
\text { Breakdowns modeled stochastically }\end{array}$ \\
\hline Model 4 & $\begin{array}{l}\text { Stochastic activity time, 24 x Hourly Pearson } \\
\text { Type IV PDF } \\
\text { Breakdowns modeled stochastically }\end{array}$ \\
\hline
\end{tabular}

Table 3: Descriptions of experimental discrete event simulation models.

The basic model used in these experiments was developed by the host organization during the design of the system. The system is a large scale assembly line, comprised of several hundred tasks, both manual and automated. To prepare this model for experimentation, the logic and layout of the model was first checked against that of the real system. The physical structure of the line was mapped and compared to the original model. Minor alterations were made to the model to incorporate elements added since the systems inception. Testing of the revised model was then conducted to ensure that the model was an acceptable representation of the existing system. To aid validity, this work was conducted jointly by the research team and the simulation expert of the host organization responsible for the building of the original model. The revised DES model was then redeveloped to incorporate the changes outlined in table 3 .

The experimentation protocol was considered to be of utmost importance to ensure that the results obtained were reliable. These protocol will determine the number of runs, length of runs, warm-up period, random number management and analysis procedure. A number of authors have proposed methods of assessing the required protocol for simulation experiments, e.g. Carrie [46]; Law and Kelton [9]; Shannon [47]. Those proposed by Fishman [48] utilize a pragmatic combination of statistical and graphical techniques and are the ones adopted here. Fishman describes the problem of designing a simulation protocol as the control of systematic and sampling errors. He defines the two types of error thus;

"Random input induces sampling error, and the dependence among observations often enhances its severity. Systematic error arises from the dependence of the observations on the initially chosen state or conditions in the simulation".
If only one replication is used, the presence of sampling error prevents the detection of systematic error. The process begins by conducting tests to define the number of replications of each simulation required to attain acceptable confidence limits. To alleviate the effects of starting conditions all the replications are started from the 'system empty' condition. The run length and warm-up periods are then selected using sensitivity testing. A full description of the methods used is given by Mason [49]. Each of the simulation models was run for 5 replications of 300 simulated shift periods, equivalent to 20 weeks of production. Each random number stream (RNS) affecting the HPV calculations within the models were changed for each replication. The RNS for the breakdown calculations were kept constant for all replications. Hence, the variation in throughput shown by the models is due to HPV alone.

The simulation experiments each produced principal performance figures representing the average production per shift and a confidence limit for the mean. The results are shown in table 4 and again graphically in figure 5 . To ensure anonymity the results are expressed as a percentage of the target performance of the system.

\begin{tabular}{|c|c|c|}
\hline $\begin{array}{l}\text { Model } \\
\text { Name }\end{array}$ & 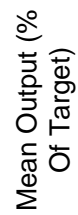 & 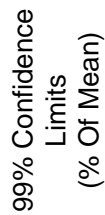 \\
\hline Model 0 & 100.0 & \pm 0.0 \\
\hline Model 1 & 87.9 & \pm 1.0 \\
\hline Model 2 & 88.1 & \pm 2.0 \\
\hline Model 3 & 86.8 & \pm 0.6 \\
\hline Model 4 & 87.3 & \pm 1.1 \\
\hline
\end{tabular}

Table 4: Mean and $99 \%$ confidence limits for simulation model results.

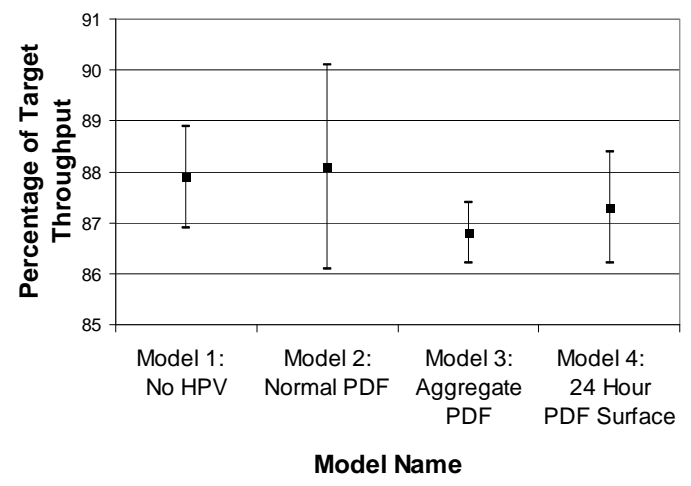

Figure 5: Graph of mean and $99 \%$ confidence limits for simulation model results. 


\section{ANALYSIS AND DISCUSSION}

When first considering the results gained from experimenting with the different models (Table 3 ), the Model 1 can initially be considered as the datum. Here there is no HPV. Against this, the Normal PDF model (Model 2) shows a doubling in the level of variation in the results though the mean is much less affected. The ranges of results from the two proposed HPV models, (Models 3 and 4) are lower than that of the 'Normal' and 'No HPV' models. Hence, incorporating HPV does cause the model to be more conservative. Although, this decrease is of the order of only $1 \%$ of the target output for the system in question, in practice this represents a very significant financial loss.

The positively skewed PDF of Model 3 is particularly interesting. This reduces the range of variation in the mean throughput, relative to both Models 1 and 2, while simultaneously reducing output. This result is counterintuitive as any increase in the spread of activity times could be expected to increase the range of mean throughput. A similar result is noted for Model 4, the PDF surface, although the range of uncertainty is larger in this case. The single PDF of Model 3 is significantly easier to apply in DES than the PDF surface of Model 4. The improved fidelity of Model 4 does not warrant the extra effort and resources required in modelling. It is recommended therefore that in most systems a single aggregated PDF model of HPV is sufficient.

The expectation of the host organization was that activity time HPV would have a large impact on performance, although this is not supported by the simulation results. The difference between Models 0 and 1 is of the order of $12 \%$ of the target throughput. Thus, in this case, the stochastic modelling of breakdowns has a much larger impact on system throughput than HPV models [50]. It is valuable to question why HPV does not have the expected impact on the performance of this system. The system under study has over 200 separate manual and automated operations, and hence exhibits very complicated behaviour. The simulations clearly illustrate that in its normal mode of operation there is a rather intermittent flow within the system. For example, under normal operating conditions the assembly line appears to be separated into a number of areas, almost cellular in nature, where constant material flow occurs separated by full buffers. Products appear to flow between these areas of the line in "pulses". The location of the 'cells' changes over time as breakdowns, shortages etc, occur. Hence, the inclusion of even relatively large time variations on operations is unlikely to have much effect, as in this instance system performance is dominated by the dynamics of the system itself. Such behaviour has been noted in previous studies [20].

To further explore the impact of HPV in systems where the dynamics of the system are less dominant, a number of small hypothetical systems were modelled with between one and four serial operations joined by conveyors. The models were run with no HPV present, and then repeated with the aggregated PDF distribution used in Model 3 in the previous tests. The results are shown in figure 6. Each model was run with a number of different planned activity times, ranging from 0.31 units to 2 units. The cycle time was constant at 1 unit for all models. In the case of a single isolated operation, the difference between throughput from the HPV and non-HPV models is of the order of two to three percent for all the planned activity times. As the number of stations increases, the difference increases, with increasing activity time, to a maximum of $23 \%$, at an activity time of 1 unit, and then decreases with further increases in activity time.

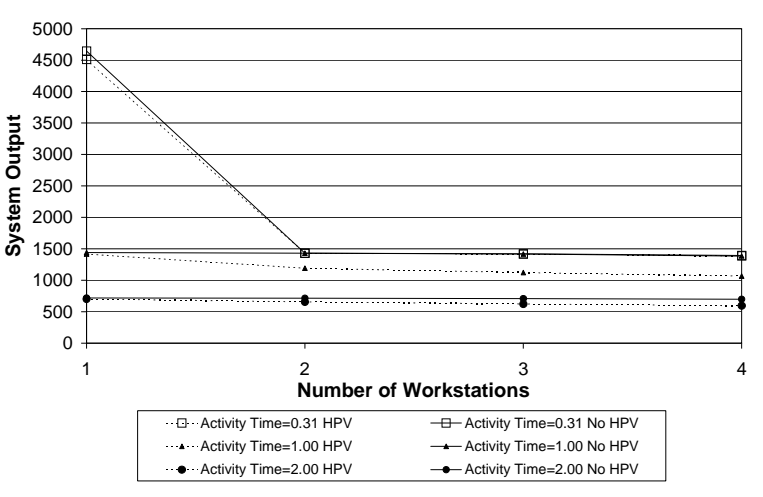

Figure 6: Output response of conceptual models with 1-4 operations with and without HPV

This maximum decrease due to HPV occurs at a planned activity time value which, due to the interaction with other system parameters, i.e. the conveyor cycle time, leads to a dramatic increase in the magnitude of the effect of HPV. Hence, when dealing with larger models with complex system dynamics it is difficult to predict whether the inclusion of HPV will have a significant effect or not.

\section{CONCLUSIONS AND FUTURE WORK}

To summarize the results therefore, it is clear that although the HPV distribution approach does result in a reduction in DES model output, in some systems it is not significant when compared to the loss in productivity caused by other disruptions. It is also clear that some systems, by combination of critical parameters, may be more prone to the impacts of HPV than others. Hence, testing for sensitivity to HPV is an important consideration. 
In particular, it has been shown that, in the system under study, the best theoretical distribution to represent empirical HPV is the Pearson Type IV. This is contrary to the conclusions of Knott and Sury [22], and other earlier work in this area.

In terms of further work, the type IV distribution is a very flexible mathematical form, and the shape of the resulting curve is governed by a number of parameters. Unlike the Normal distribution, which can be defined by its mean, magnitude and standard deviation, many more parameters must be defined to create a type IV curve that is characteristic of HPV in a given context. Hence, relatively large datasets are required when gathering HPV data for this purpose. Further work is required to examine the relationships between these "shape" parameters and task factors, in order to define generic HPV distributions which would be widely applicable. In addition, further studies in different task contexts are required to verify the generic nature of HPV.

The current research has concentrated solely on the impact of HPV in terms of activity time. To fully assess the importance of HPV it is necessary to extend the models of HPV to include error rates, and both corrective and disruptive behavior, and in doing so it is anticipated that worker HPV will be found to have a larger impact on system performance.

\section{REFERENCES}

[1] McCarthy, B. and Wilson, J.R. 2001, Human Performance in Planning and Scheduling. Taylor and Francis.

[2] Goldhar, J.D. and Jelinek, M. 1983, Plan for economies of scope. Harvard Business Review, November-December, 141-148.

[3] Klingstam, P. and Gullander, P. 1999, Overview of simulation tools for computeraided production engineering. Computers in Industry 38, 173-186.

[4] Holst, L. and Bolmsjö, G. 2001, Simulation integration in manufacturing system development: a study of Japanese industry. Industrial Management and Data Systems 101, 7, 339-356.

[5] Cunha, P., Dionisio, J. and Henriques, E. 2003, An architecture to support the manufacturing system design and planning. International Journal of Computer Integrated Manufacturing 16, 7-8, 605-612.
[6] Shingo, S. 1989, A study of the Toyota Production System from an Industrial Engieering Viewpoint, Cambridge MA, Productivity Press.

[7] Weston, R.H., Clements, P.E., Shorter, D.N., Carrott, A.J., Hodgson, A. , and West, A.A. 2001, On the explicit modelling of systems of human resources, International Journal of Production Research, 39, 2, p.185-204.

[8] Simon, H.A. 1990, Prediction and Prescription in Systems Modeling, Operations Research, 38, 1 p.7-14.

[9] Law, A.M. and Kelton, W.D. 1991, Simulation Modelling and Analysis, McGrawHill.

[10] Chan, F.T.S. and Jiang B. 1999, Simulationaided design of production and assembly cells in an automotive company. Integrated Manufacturing Systems 5, 10, 276-283.

[11] Baines, T.S. and Kay, J.M. 2002, Human performance modeling as an aid in the process of manufacturing system design: a pilot study. International Journal of Production Research 40, 1, 2321-2334.

[12]Ball, P. 1998, Abstracting performance in hierarchical manufacturing simulation. Journal of Materials Processing Technology 76, 246-251.

[13]De Souza, R. and Zhao, Z.Y. 1997, A case for intelligent representation of dynamic resources in simulation. International Journal of Production Research 35, 12, 3289-3302.

[14] Checkland, P.B. (1981) Science and the systems movement. In: Open University Systems Group, Systems Behavior, 26-43.

[15] Kalpakjian, S. and Schmid, S.R. 2001, Manufacturing Engineering and Technology. New Jersey: Prentice Hall.

[16]Ehrhardt, I. Herper, H. and Gebhardt, H. 1994, Modelling strain of manual work in manufacturing systems. Proceedings of the 1994 Winter Simulation Conference. IEEE.

[17]Freudenberg, R. and Herper, H. 1998, Simulation of workers in manufacturing systems. Proceedings of the 1998 Winter Simulation Conference, Volume 2. IEEE. 
[18] Wilson, J.R. Neely, A.D., Aggarwal, N. 1993 Allowing for the human element - human factors in small manufacturing enterprises. International Journal of Human Factors in Manufacturing, 3, 2, 193-205.

[19]Dietrich, B.L. 1991, A taxonomy of discrete manufacturing systems. Operations Research 39, 6, 886-902.

[20] Slack, N.D. and Wild, R. 1980, The nonsteadystate performance of unpaced manual assembly lines, International Journal of Production Research, 5,583-595.

[21]Taylor, F. W. 1917, The Principles of Scientific Management, Harper and Brothers, New York.

[22] Knott, K. and Sury, R.J. 1987, A study of work-time distributions on unpaced tasks, IIE Transactions, March, 50-55.

[23] Conrad, R. 1954, The rate of paced manmachine systems, Journal of the Institution of Production Engineers, 33, 10, 562.

[24] Dudley, N.A. 1955, Output Pattern in Repetitive Tasks, PhD Thesis, University of Birmingham.

[25] Dudley, N.A. 1963, Work Time Distributions, International Journal of Production Research, 2, 2, 137-144.

[26] Murrell. K.F.H. 1962, Operator variability and its industrial consequences, International Journal of Production Research, 1, 2, 39-55.

[27] Sury, R.J. 1964, An industrial study of paced and unpaced operator performance in a single stage work task, International Journal of Production Research, 3, 2, 91-103.

[28] Tempelmeier, H. 2003, Practical considerations in the optimization of flow production systems, International Journal of Production Research, 41, 1, 129-170.

[29] Mason, S., Siebers, P-O., Baines, T., Ladbrook, J. 2002, Humans: The missing link in simulation. OR Simulation Study Group Two-Day Workshop, 20-21 March, University of Birmingham. Proceedings edited by Eldabi, T., Robinson, S., Taylor, S. J. E. and Wilcox, P. A.

[30] Baines, T., Mason, S., Siebers, P-O., and Ladbrook, J. Humans: The missing link in simulation. Simulation Practice and Theory. 12 , issue 7-8, 515-526.

[31] Dudley, N.A. 1960, The effect of pacing on operator performance, International Journal of Production Research, 1, 2, 60-72.

[32] Buffa, E.S. 1987. Modern production operations management, John Wiley, pp.642.

[33] Baines, T., Hadfield, L., Mason, S., and Ladbrook, J. 2003, Using empirical evidence of variations in worker performance to extend the capabilities of discrete event simulations in manufacturing. Winter Simulation Conference, New Orleans, 7-10 December 2003. Proceedings edited by Chick, S., Sanchez, P. J., Ferrin, D. and Morrice, D. J.

[34] Gilbertova, S. and Benes, V. 1970, Criteria on evaluation of monotony of work, International Journal of Production Research, 8, 4, 307-314.

[35] Roe, R.A. 1999, Work performance: a multiple regulation perspective, In: International Review of Industrial and Organizational Psychology, 14, 231-335 ed. Cooper, C.L. and Robertson, I.T. John Wiley.

[36] Sury, R.J. 1971, Aspects of assembly line balancing, International Journal of Production Research, 9, 4,501-512.

[37] Anderson, D. R. and Moodie, C. L. 1969, Optimal buffer storage capacity in production line systems, International Journal of Production Research, 7, 3, 233-240.

[38] Liu, C.-M., Su, S.-F. and Lin, C.-L. 1996, Predictive models for performance evaluation of serial production lines, International Journal of Production Research, 34, 5, 12791291.

[39] Buxey, G. 1979, The nature of manual, moving belt flowlines with overlapping stations, International Journal of Production Research, 17, 2, 143-154.

[40] Knott, A.D. 1970, The inefficiency of a series of work stations - a simple formula, International Journal of Production Research, 8, 2, 109-119.

[41] Ghosh, S. and Gagnon, R. J. 1989, A comprehensive literature review of the design balancing and scheduling of assembly systems, International Journal of Production Research, 27, 4, 637-670. 
[42] Erel, E. and Sarin, S.C. 1998, A survey of the assembly line balancing procedures, Production Planning and Control, 9, 5, 414434.

[43] Minors, D.S. and Waterhouse, J.M. 1981, Circadian Rhythms and the Human. Wright.

[44] Czeizler, C.A. 1978, Human Circadian Physiology: Internal Organization of Temperature, Sleep-Wake and Neuroendocrine Rhythms Monitored in an Environment Free of Time Cue. PhD Thesis, Stanford University.

[45] Ladbrook, J. 2003, Personal Communication.

[46] Carrie, A. 1988, Simulation of Manufacturing Systems, John Wiley.

[47] Shannon, R.E. 1975, Systems Simulation, Prentice Hall.

[48] Fishman, G.S. 1978, Principles of Discrete Event Simulation, John Wiley and Sons.

[49] Mason, S.A. 2005, Stochastic and Temporal Modelling of Human Performance Variation in Flow Line Assembly Systems. PhD Thesis, Cranfield University.

[50] Hadfield, L., Baines, T. and Ladbrook, J. An empirical analysis of assembly line disruption: implications for discrete event simulation. In preparation.

\section{ACKNOWLEDGEMENTS}

The authors would like to thank the referees for their constructive critique of this paper.

\section{AUTHOR BIOGRAPHIES}

STEVE MASON received a PhD from Cranfield University in 2005. His experience includes industrial project engineering, research and academic roles. He is currently a postdoctoral researcher at the Centre for Business Performance, Cranfield University, investigating systems approaches to organisational performance.

TIM BAINES is the Professor of Strategic Manufacture, JOHN KAY is the Professor of Manufacturing Systems and JOHN LADBROOK is a Visiting Fellow. All three are within the Department of Manufacture at Cranfield and specialise in manufacturing systems design and modelling. The corresponding author is Tim
Baines and he can be reached at <t.s.baines@cranfield.ac.uk>. 